\title{
Rain-Induced Increase in Background Radiation Detected by Radiation Portal Monitors
}

\author{
R. J. Livesay, C. S. Blessinger, T. F. Guzzardo, P. A. Hausladen \\ Oak Ridge National Laboratory, 1 Bethel Valley Road, Oak Ridge, TN 37831
}

\begin{abstract}
A complete understanding of both the steady state and transient background measured by Radiation Portal Monitors (RPMs) is essential to predictable system performance, as well as maximization of detection sensitivity. To facilitate this understanding, a test bed for the study of natural background in RPMs has been established at the Oak Ridge National Laboratory. This work was performed in support of the Second Line of Defense Program's mission to detect the illicit movement of nuclear material. In the present work, transient increases in gamma ray counting rates in RPMs due to rain are investigated. The increase in background activity associated with rain, which has been well documented in the field of environmental radioactivity, originates from the atmospheric deposition of two radioactive daughters of radon-222, namely lead-214 and bismuth-214 (henceforth ${ }^{222} \mathrm{Rn},{ }^{214} \mathrm{~Pb}$ and ${ }^{214} \mathrm{Bi}$ ). In this study, rainfall rates recorded by a co-located weather station are compared with RPM count rates and High Purity Germanium spectra. The data verifies these radionuclides are responsible for the dominant transient natural background fluctuations in RPMs. Effects on system performance and potential mitigation strategies are discussed.
\end{abstract}

\section{Introduction}

The effect of radioactive precipitation has been observed at hundreds of sites around the world and extensively studied for several decades. The subject of radioactive precipitation is of interest to numerous applications that depend on environmental radiation measurements, including monitoring for environmental contamination at nuclear facilities as well as exploiting radon progeny as natural tracers to study physical processes [2]. Regardless of the application, the predominant sources of naturally occurring radioactivity in precipitation are the ${ }^{222} \mathrm{Rn}$ progeny ${ }^{214} \mathrm{~Pb}$ and ${ }^{214} \mathrm{Bi}$ that are scavenged within clouds and deposited on the ground during rain [9].

The present work is focused on characterizing the response of Radiation Portal Monitors (RPMs) to rain-induced increases in background gamma-ray radiation to ultimately maximize system performance. RPMs typically consist of two gamma ray detectors, each consisting of a slab of polyvinyl toluene (PVT) scintillator, and two neutron detector modules, consisting of ${ }^{3} \mathrm{He}$ proportional counters moderated by polyethylene, mounted in an upright rectangular pillar. The RPMs target passive detection of the low energy gamma-ray emissions of special nuclear material (SNM) in the PVT and neutrons of a broad energy in the ${ }^{3} \mathrm{He}$ proportional counters. Typically, two pillars are arranged to face each other, one on either side of a lane of commercial traffic at a port or border crossing. RPMs record "gross counts" corresponding to gamma rays with energies between an upper and lower level discriminator. The values of these discriminators are determined programmatically to target specific threat materials. The data is displayed as the counts that fall in this window as a function of time (see Figure 1 and Figure 2). The background is generally expected to be constant, but as can be seen from the data there are 
ongoing deviations from the average, some of which are significant. The study of these deviations gives information about the health of the systems.

The Office of the Second Line of Defense has deployed an extensive array of RPMs to deter the transport of illicit nuclear material, as part of a larger nuclear non-proliferation effort. RPMs are currently operating in hundreds of sites around the world. Some are used indoors to monitor pedestrian traffic while others monitor the passage of cargo containers through seaports and border crossings.

During decay by means of their most probable branches, ${ }^{214} \mathrm{~Pb}$ and ${ }^{214} \mathrm{Bi}$ emit gamma-rays of $351.99 \mathrm{keV}$ and $609.32 \mathrm{keV}$, respectively. This emission of radiation, and the subsequent increase in background counting rate, is easily observed by using conventional radiation detectors. In addition to the energy of gamma-rays, the characteristic decay time can be used for isotope identification: ${ }^{222} \mathrm{Rn}$ decays with a half life of 3.8 days while ${ }^{214} \mathrm{~Pb}$ and ${ }^{214} \mathrm{Bi}$ decay with much shorter half lives of 27 minutes and 20 minutes, respectively. This study utilizes these physical properties of radon progeny to isolate the source of the background increases observed in RPMs.

In this work, "background" will be used to refer to the signal measured by an RPM, while not occupied, and the "static background" will refer to the steady-state portion of the background. The rain-deposition of radioactive nuclei provides a transient source term to the static background, yielding the measured background. In the case of RPMs, the dominant source of background is typically due to construction materials, soil, and bedrock (which contain ${ }^{40} \mathrm{~K}$ and $\left.{ }^{238} \mathrm{U}\right)$.

Radioactive sources travel near and through RPMs for a variety of reasons. Normally occurring radioactive material (NORM), medical treatments, orphaned industrial sources, and weather all contribute to the signal measured by RPMs. Arguably the most common deviation from the natural radioactive background is due to the deposition of radioactive ${ }^{214} \mathrm{Bi}$ and ${ }^{214} \mathrm{~Pb}$ from precipitation. This effect is seen in varying degrees in most outdoor RPMs, with some sites experiencing background fluctuations of more than $100 \%$ of their average background values. The effect appears as positive spikes in the data, since the decay time is very short (hours) compared to the scale over which data is generally analyzed (months), see Figure 1. When the scale is expanded for more detailed analysis of the spikes, the structure of the effect becomes visible. The structures have a variety of shapes and durations, but all have a characteristic halflife of approximately 30 minutes. Short rains are the easiest to identify because they possess a short rise time and relatively long decay time. For short rains, the background will be elevated above the nominal background for several half-lives which often amounts to around 4 hours. The decay shape is a consequence of the quick deposition of radionuclides and their decay. More complicated shapes are due to multiple rains occurring within the decay time, increasing the activity with each deposition, causing a rough saw-tooth pattern. 


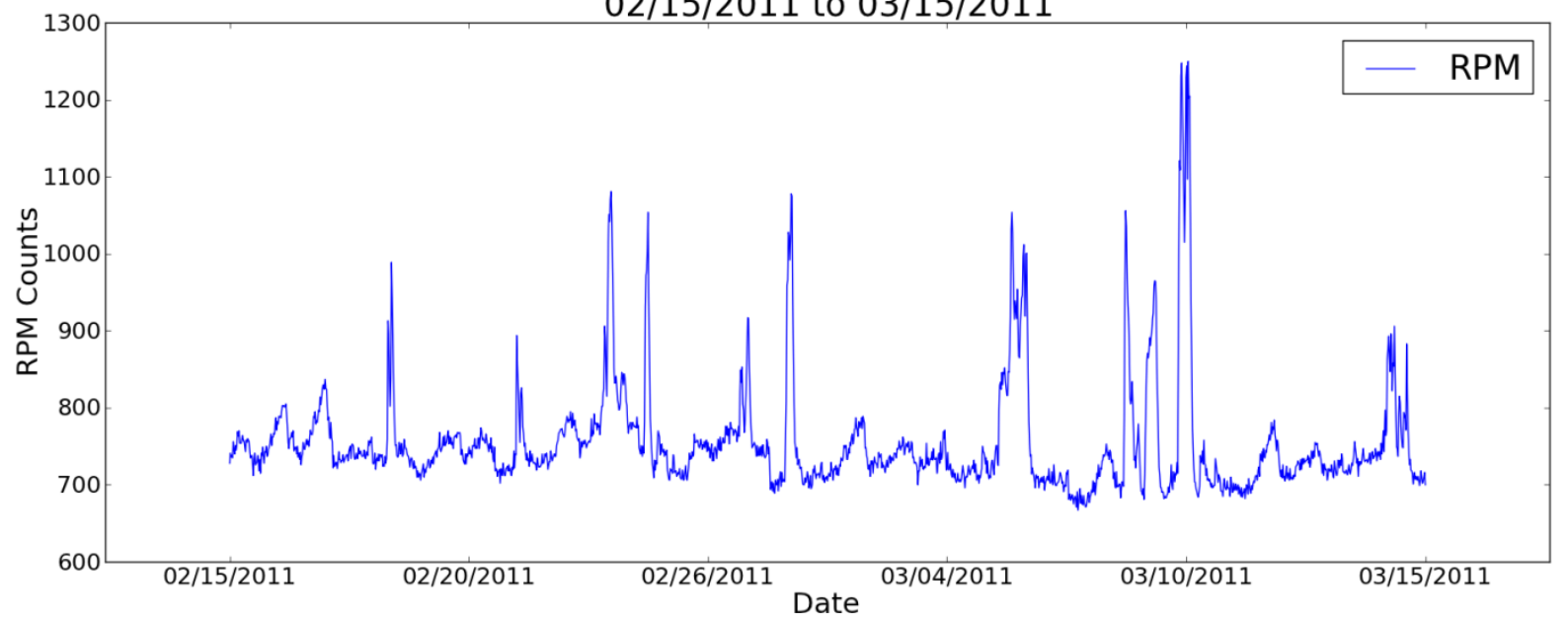

Figure 1: One-month window of background data from one RPM (sum of individual countrates of four gamma detectors). The spikes in the data are clear deviations from the static background (well outside the statistical variance). Further analysis shows the same spikes are seen in all the RPMs at this site.

Although the effect of rain dissipates in a few hours, RPMs underperform until the additional background radiation decays away and the background returns to the expected background value used to calculate system performance. The rain-induced increase in background affects the statistical precision of measurements and, as such, should be addressed, alongside other challenges present in the deployment and operation of RPMs. Any increase in background is detrimental to RPM performance because as the background increases, sensitivity of the RPM decreases. During periods of increased background, a higher activity source is required to trigger an alarm due to the dependence of the alarm algorithm on the standard deviation of the background.

\section{Current Work}

In order to characterize and ultimately mitigate the effects of rain-induced background increases observed in RPMs, the Portal Monitor Test Bed was constructed at Oak Ridge National Laboratory. The experimental test bed features an ORION 510 Weather Station mounted on a small instrument trailer, a RPM, and a high purity germanium detector. Data streams from the detection devices are combined and analyzed with an in-house code specifically designed for the measurement of radioactive decay. Time stamps from the acquisition computers provide the necessary correlation between meteorological and radiological events. A detailed study of approximately 50 rain events support the basic model of radon transport which relies on the deposition of radon progeny through precipitation. Establishing the basic mechanism for the rain-increased background in RPMs is a first step in determining suitable remediation solutions.

Gamma-rays with energies on the order of a few hundred keV are most likely to interact in PVT detectors through the process of Compton scattering. This process only transfers a fraction of a gamma-ray's energy into scintillated light resulting in a mostly featureless spectrum (Figure 3). 
Therefore, it is difficult to determine the initial energy of an incident gamma-ray detected by PVT.

The process is complicated further by the fact that currently deployed RPMs use a low-energy window in attempt to alarm on special nuclear material and not NORM. This is important for the study of the radioactive precipitation because the $351.99 \mathrm{keV}$ and $609.32 \mathrm{keV}$ gamma-rays from ${ }^{214} \mathrm{~Pb}$ and ${ }^{214} \mathrm{Bi}$ will only be detected in an RPM if scattering events occur that reduce the energy of the gamma-ray within the established energy window. As a result, it is difficult to directly identify the decay of ${ }^{222} \mathrm{Rn}$ progeny in RPM data and link detection events to radioactive precipitation. However, in contrast to the gamma response of PVT detectors, the resolution of high purity germanium (HPGe) detectors allows the precise measurement of gamma-ray energy. By using a HPGe, the decay of ${ }^{214} \mathrm{Lead}$ and ${ }^{214}$ Bismuth can be directly measured.

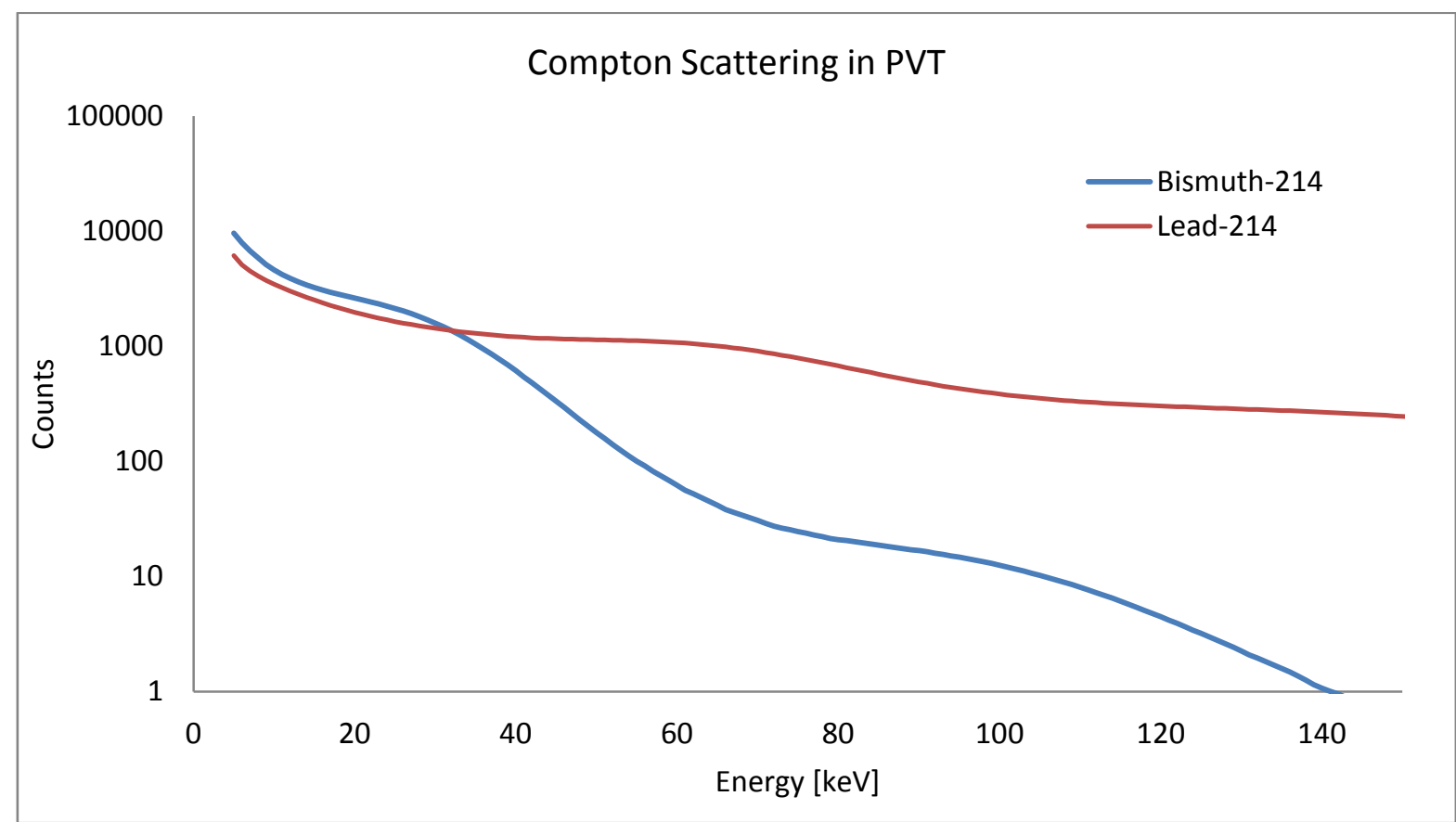

Figure 2: PVT response to ${ }^{214} \mathrm{~Pb}$ and ${ }^{214} \mathrm{Bi}$. The initial ratio of radon-daughter concentrations are taken from literature [2]. The detection efficiency is modeled using GADRAS, and the solid angle of the detectors is calculated using MCNP. The increased background is assumed to be entirely due to the addition of Lead and Bismuth, although there are small contributions from other radioactive species [7].

A line of reasoning sometimes used to explain the background increase in RPMs is that the atmospheric pressure decreases before precipitation which allows more radon to exhaust from the ground. The data presented in this work shows this effect is negligible over the time frame of average rain events. This result is founded on the observation that the half-life of the background decay rate is approximately 30 minutes, inconsistent with the 3.8 day half-life of ${ }^{222} \mathrm{Rn}$. This demonstrates that ${ }^{214} \mathrm{~Pb}$ and ${ }^{214} \mathrm{Bi}$ are being directly deposited on the ground after decaying from ${ }^{222} \mathrm{Rn}$ present in the cloud. If the background increase was due to ${ }^{222} \mathrm{Rn}$ exhausting from the ground during precipitation, the decay of ${ }^{222} \mathrm{Rn}$ into ${ }^{214} \mathrm{~Pb}$ and ${ }^{214} \mathrm{Bi}$ would 
occur over many 3.8 day half-lives. Furthermore, the decay of ${ }^{214} \mathrm{~Pb}$ and ${ }^{214} \mathrm{Bi}$ would also occur over multiple days.

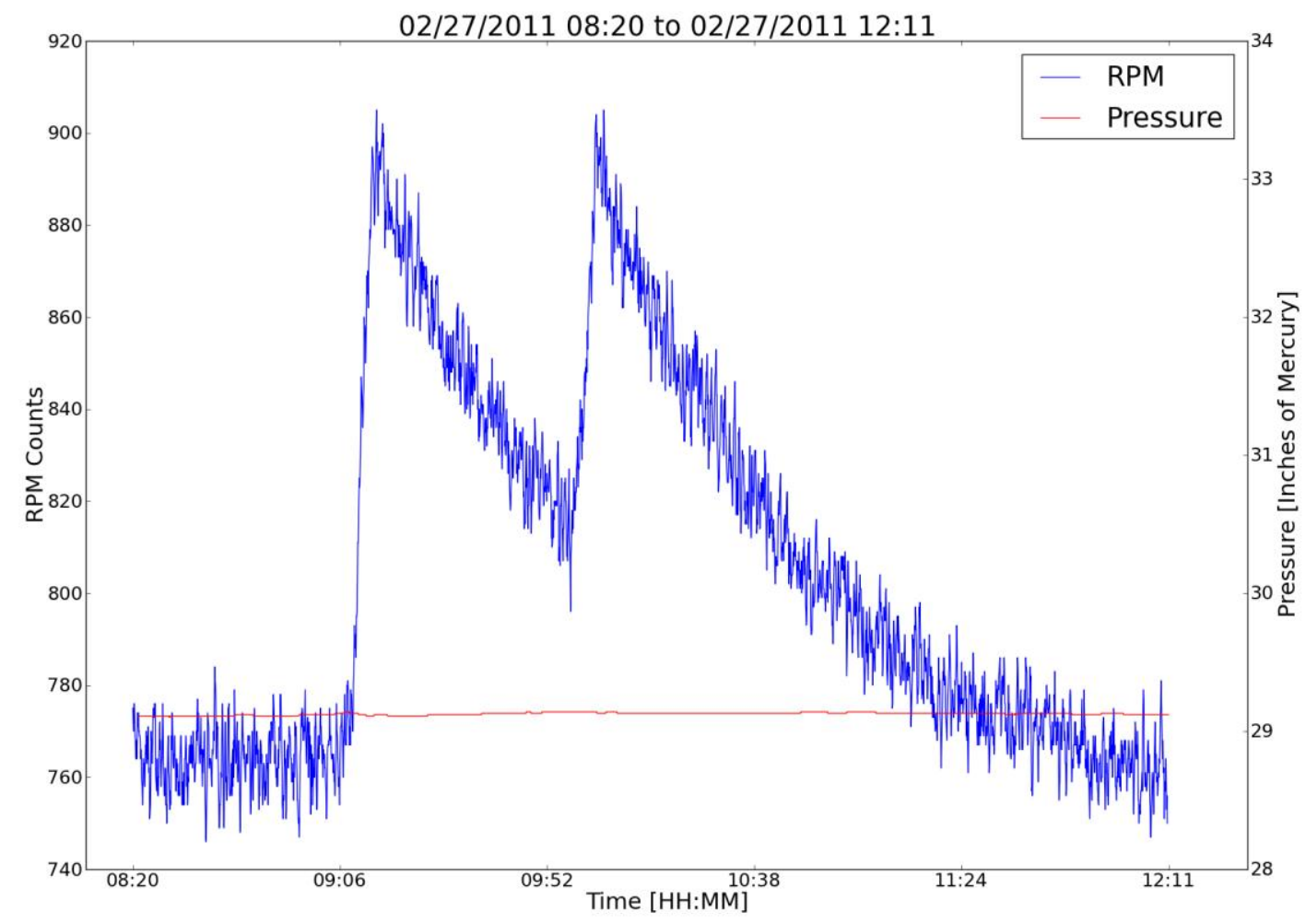

Figure 4: RPM response and pressure plotted against time to show the non-correlation of the two variables. 


\section{Isotopic Response from HPGe}

To explicitly show the decay of ${ }^{214} \mathrm{~Pb}$ and ${ }^{214} \mathrm{Bi}$ as well as their contribution to the RPM response, spectra were recorded for several rain-events with a HPGe detector. Regions of Interest (ROIs) were established to measure the $351.99 \mathrm{keV}$ and $609.32 \mathrm{keV}$ gamma-rays of ${ }^{214} \mathrm{~Pb}$ and ${ }^{214} \mathrm{Bi}$, respectively, to determine the presence of these two radioactive metals in the rain. There are several other prominent gamma rays from $\mathrm{Pb}$ and $\mathrm{Bi}$ : from $\mathrm{Pb}(351,295,242)$, from $\mathrm{Bi}(1764,1120,609,63)$. Figure 6 shows a HPGe spectra, one recorded during a rain event and one recorded during a period of no precipitation. The plot clearly shows the difference in the ${ }^{214}$ Lead and ${ }^{214}$ Bismuth peak with and without precipitation.
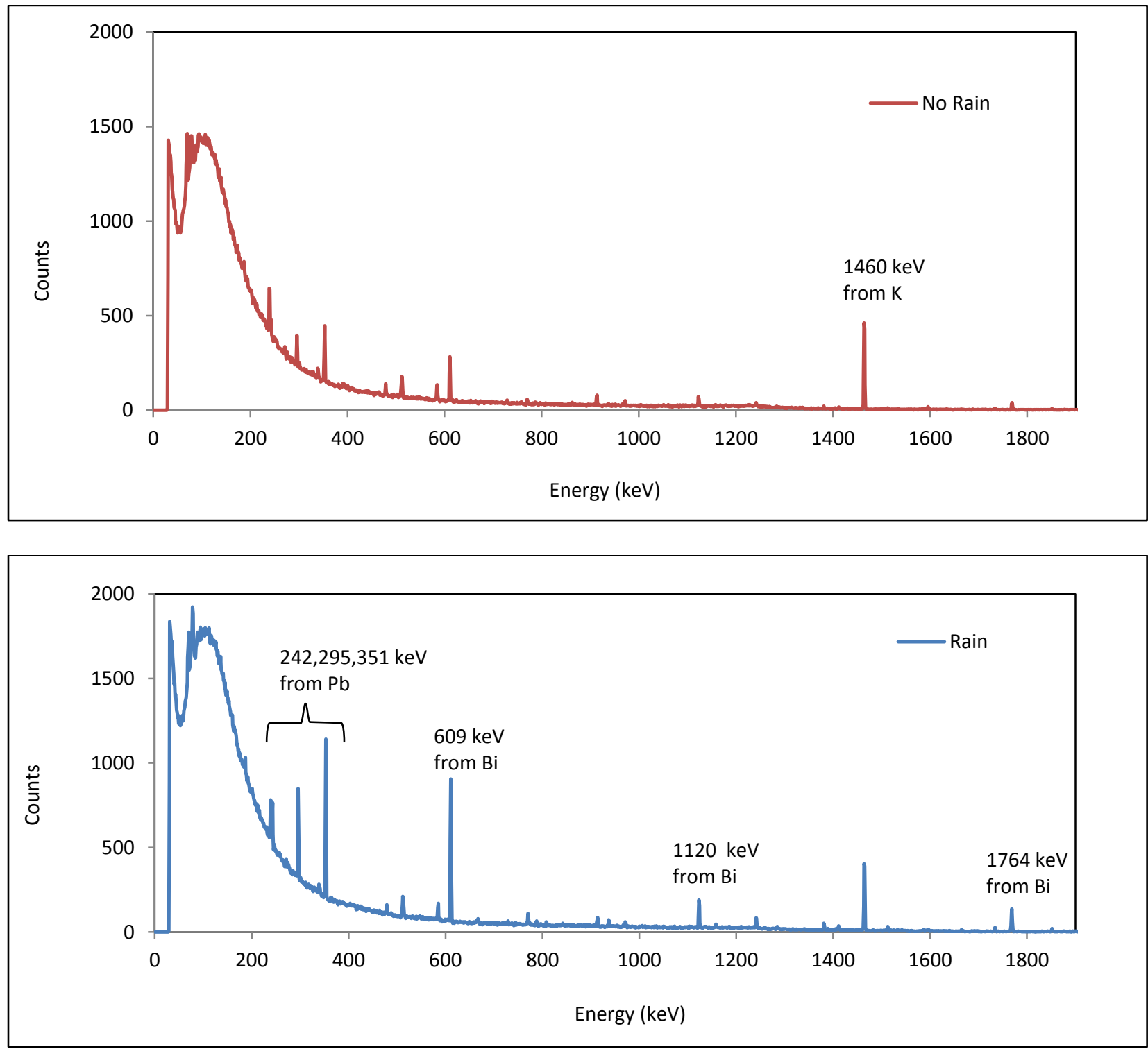

Figure 5: 300 second counts recorded with a HPGe with rain (above) and without rain (below). It's noteworthy that an increase in the gammas from $\mathrm{Pb}$ and $\mathrm{Bi}$ are measured during rain. They are already in the nominal background, without rain. 
The counts from the ${ }^{214} \mathrm{~Pb}$ and ${ }^{214} \mathrm{Bi}$ ROIs were recorded throughout the duration of several rain events. Figure 6 shows the behavior of the $\mathrm{Pb}$ and $\mathrm{Bi}$ decays over the course of the rain event, alongside the RPM response. During precipitation, the background is increased by the decay of ${ }^{214} \mathrm{~Pb}$ and ${ }^{214} \mathrm{Bi}$, but the background due to the local sources remains constant.

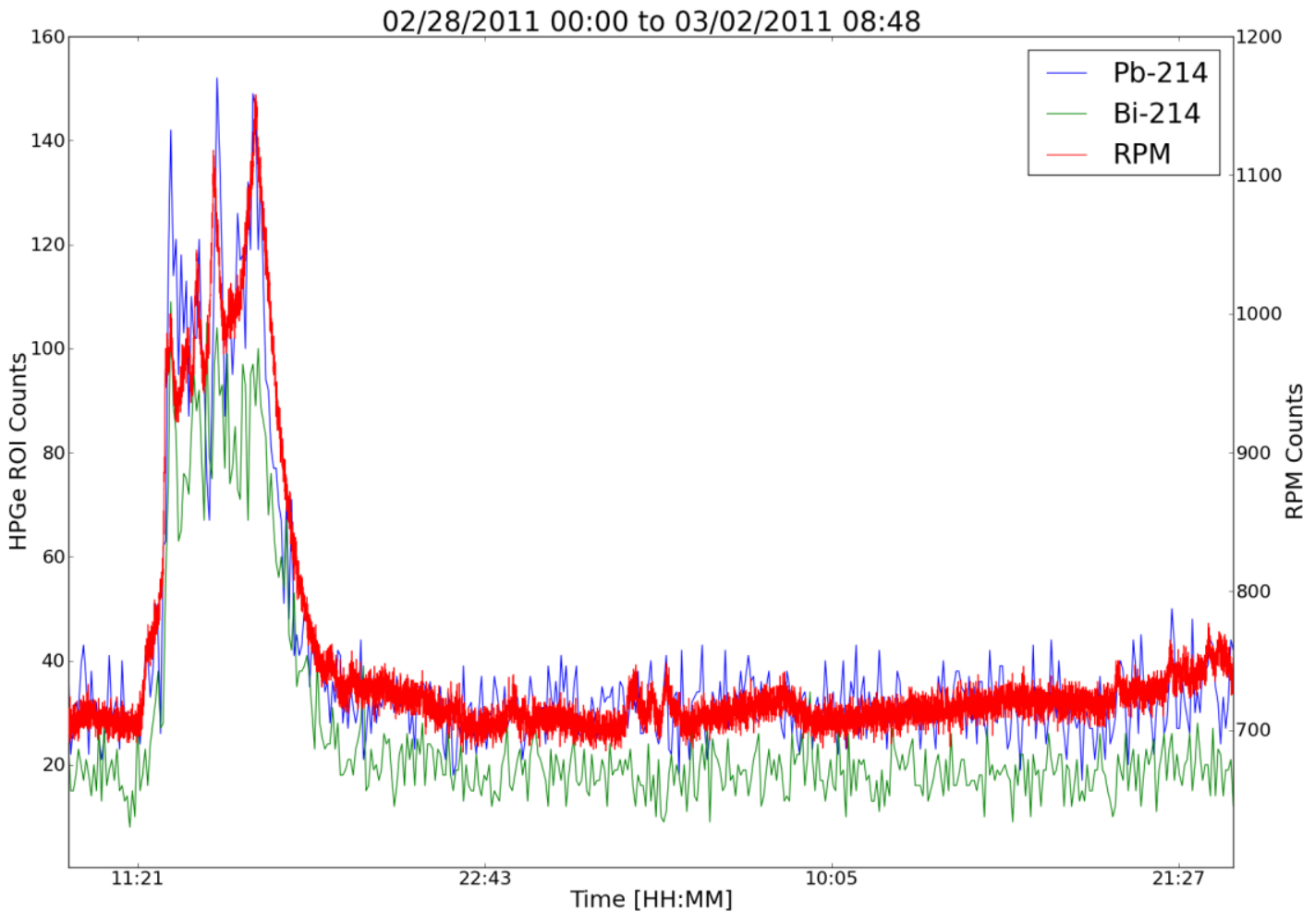

Figure 6: The decay of ${ }^{214}$ Lead and ${ }^{214}$ Bismuth are the main contributors to the rain-induced background increase in RPMs. In this figure, the $\mathrm{Pb}$ and $\mathrm{Bi}$ were counted in a HPGe detector.

\section{Formal Theory}

Due to a fundamental understanding of the background increase mechanism, the shape of the RPM response during and after a rain-event can be predicted, given the rate of rain. The mathematical prediction solely relies on the deposition of ${ }^{214} \mathrm{~Pb}$ and ${ }^{214} \mathrm{Bi}$ and the initial ratio of ${ }^{214} \mathrm{~Pb}$ to ${ }^{214} \mathrm{Bi}$, which is taken from literature in this work [2]. The dynamic behavior is taken directly from the concept of the growth of daughter activities. Since ${ }^{214} \mathrm{~Pb}$ decays to ${ }^{214} \mathrm{Bi}$, ${ }^{214} \mathrm{Lead}$ has only a decay term for each increment of rain, whereas ${ }^{214}$ Bismuth has a source term and a decay term. This model includes only the contribution from rainwater that reaches the ground.

Equation 1.1 defines the ${ }^{214} \mathrm{~Pb}$ rate of decay as a function of time from the introduction of ${ }^{214} \mathrm{~Pb}$ at time $t=0$. Equation 1.2 defines the ${ }^{214} \mathrm{Bi}$ rate of decay which is directly affected by the ${ }^{214} \mathrm{~Pb}$ rate of decay. 
Given:

1. $A_{i}(t)=$ rate of decay of isotope $i$ at time $t$

2. $A_{i}{ }^{O}=$ initial rate of decay

3. $\lambda=$ decay constant

$$
\begin{aligned}
& A_{P b}(t)=A_{P b}^{0} \cdot e^{-\lambda_{P b} \cdot t} \\
& A_{B i}(t)=A_{P b}^{0} \frac{\lambda_{P b}}{\lambda_{B i}-\lambda_{P b}} \cdot\left(e^{-\lambda_{P b} \cdot t}-e^{-\lambda_{B i} \cdot t}\right)+A_{B i}^{0} \cdot e^{-\lambda_{B i} \cdot t}
\end{aligned}
$$

To determine the count rate recorded in the RPM from equations 1.1 and 1.2, constants must be applied to account for a variety of efficiency factors. The constants account for the intrinsic efficiency of the detector, the solid angle subtended by the detector, attenuators between the source and the detector, self-attenuation, and any additional factors that contribute to the difference between the activity of the source and the count rate recorded by the detector. Equations 1.3 and 1.4 define the count rate recorded in the RPM due to an impulse of rain. The impulse response function (Equation 1.4) is shown in Figure 8 and is used with the rain intensity to predict the RPM count rate for an entire rain event.

Given:

1. $\xi_{i}=$ efficiency constant

2. $G_{i}(t)=A_{i}(t) \cdot \xi_{i}=$ recorded count rate due to impulse of rain

$$
\begin{aligned}
& G_{\text {rain }}(t)=G_{P b}(t)+G_{B i}(t) \\
& G_{\text {rain }}(t)=G_{P b}^{0} \cdot e^{-\lambda_{P b} \cdot t}+G_{P b}^{0} \frac{\lambda_{P b}}{\lambda_{B i}-\lambda_{P b}} \cdot\left(e^{-\lambda_{P b} \cdot t}-e^{-\lambda_{B i} \cdot t}\right)+G_{B i}^{0} \cdot e^{-\lambda_{B i} \cdot t}
\end{aligned}
$$

The expected count rate for an entire rain event, $M(t)$, is the convolution of $f(t)$ and $G_{\text {rain }}(t)$.

Given:

1. $f(t)=$ the rate of rain [inches / second]

2. $M(t)=$ the expected count rate from detector for rain event

3. all rain has an equal density of radon progeny

$$
\begin{aligned}
& M(t)=\int_{-\infty}^{\infty} f(t) \cdot G_{\text {rain }}(t-\tau) \partial \tau \\
& M(t)=\int_{-\infty}^{\infty} f(t) \cdot\left[G_{P b}^{0} \cdot e^{-\lambda_{P b} \cdot(t-\tau)}+G_{P b}^{0} \frac{\lambda_{P b}}{\lambda_{B i}-\lambda_{P b}} \cdot\left(e^{-\lambda_{P b} \cdot(t-\tau)}-e^{-\lambda_{B i} \cdot(t-\tau)}\right)+G_{B i}^{0} \cdot e^{-\lambda_{B i} \cdot(t-\tau)}\right] \partial \tau
\end{aligned}
$$


Equation 1.6 is used to produce a curve that is proportional to the RPM rain-response. This mathematically predicted response is proportional to the total RPM count rate with background subtracted. To bring the predicted response into agreement with the RPM response requires the determination of a normalization factor which is primarily dependent on the activity-density of the rain. In the following examples, the activity-density of the rain is arbitrarily adjusted so the magnitudes of the responses are equal.

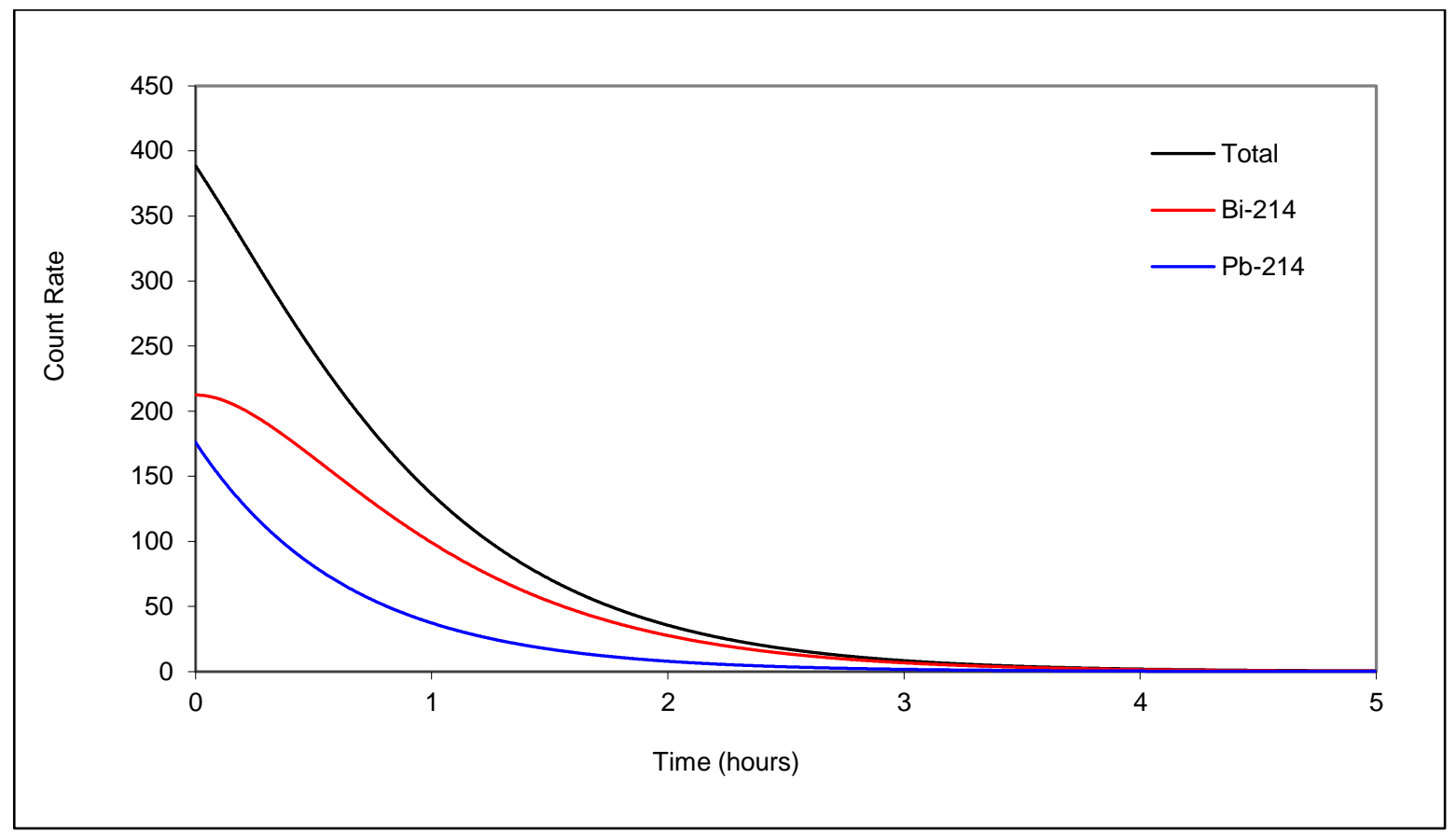

Figure 7: RPM response to $1 \mathrm{~Bq} / \mathrm{cm}^{2}$ of $214 \mathrm{Bi}$ and $214 \mathrm{~Pb}$ in secular equilibrium $\left(0.5 \mathrm{~Bq} / \mathrm{cm}^{2}\right.$ each $)$ deposited instantaneously a $\mathrm{t}=0$. The sum is measured by the RPM.

Figure 8 shows the simplicity of fitting a "delta function" rain, a rain event that is short compared to the half lives of ${ }^{214} \mathrm{~Pb}$ and ${ }^{214} \mathrm{Bi}$. Figure 9 shows the proficiency of this method at describing complicated RPM behavior. However, there are some obvious challenges to this method. Firstly, there is some natural fluctuation in the natural radiation background which in turn leads to some disagreement, and the variability of the volumetric activity of each precipitation event further contributes to the disagreements. 
(a)

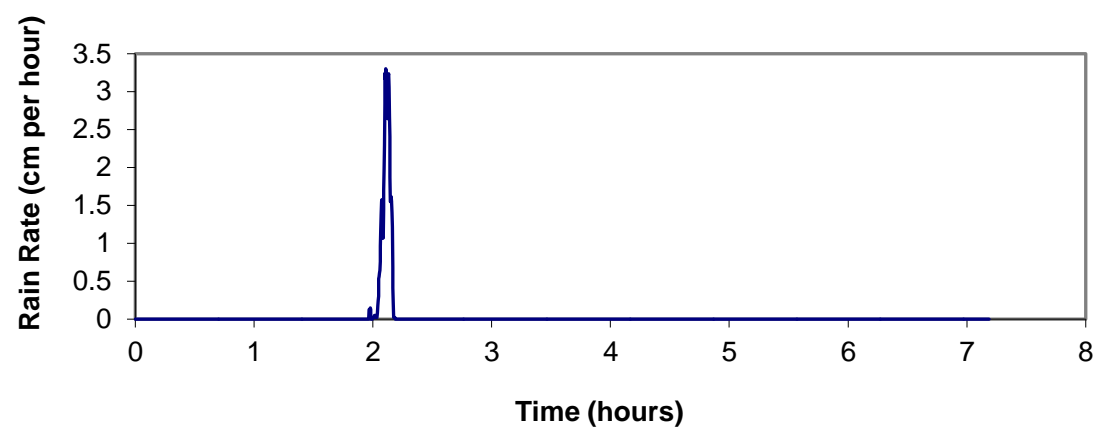

(b)

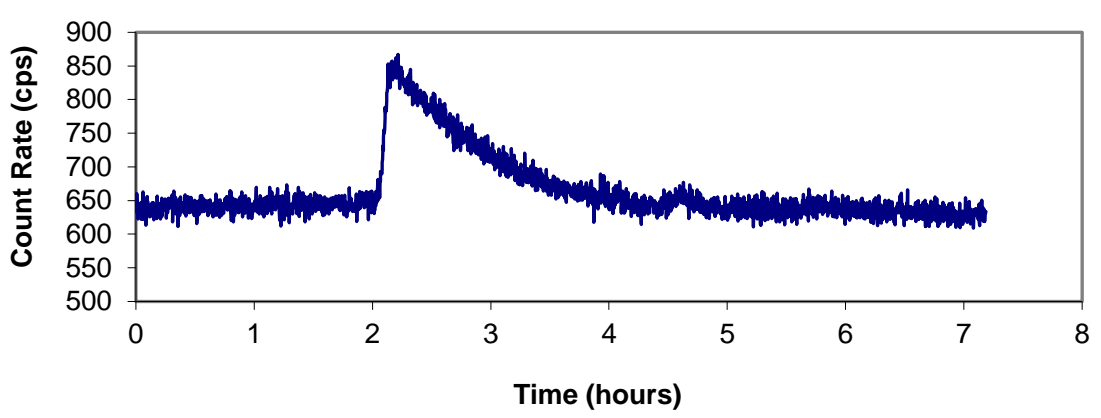

(c)

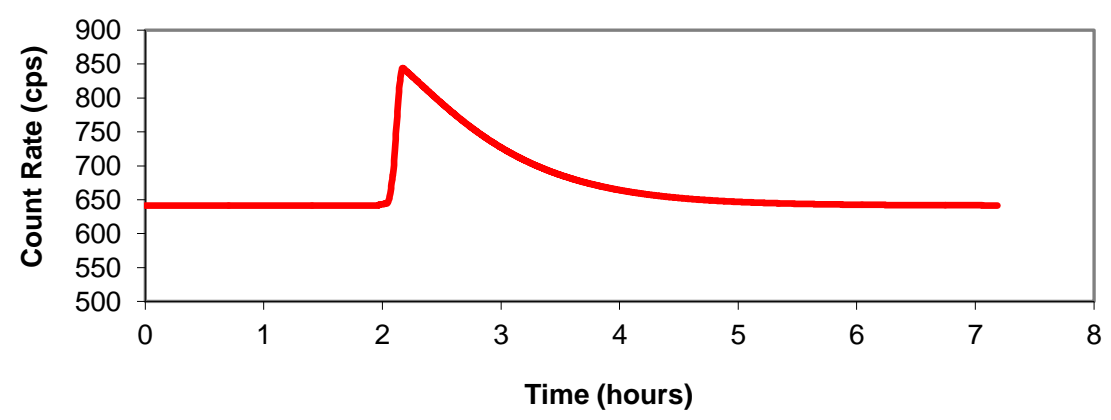

(d)

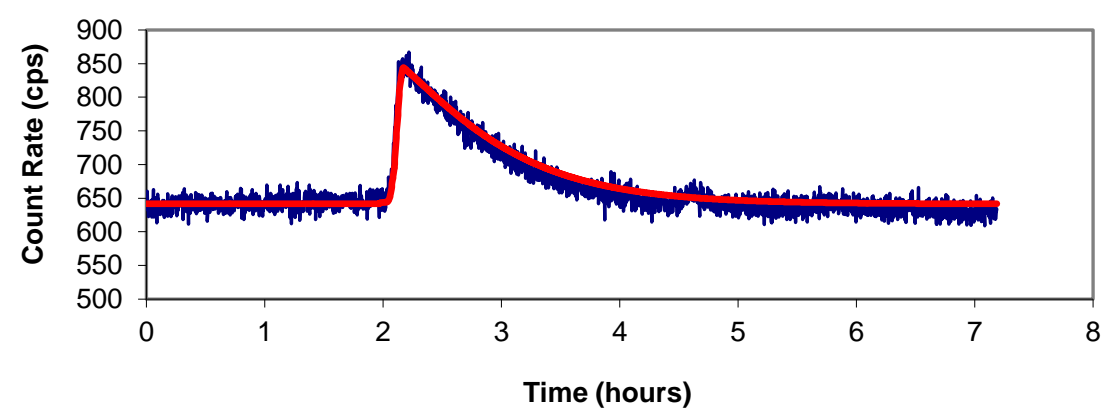

Figure 8: (a) rate of rain in $\mathrm{cm} / \mathrm{hr}$ for an isolated rain event, (b) RPM response to the rain, (c) mathematically predicted response, (d) agreement between the actual RPM response and the predicted response 
(a)

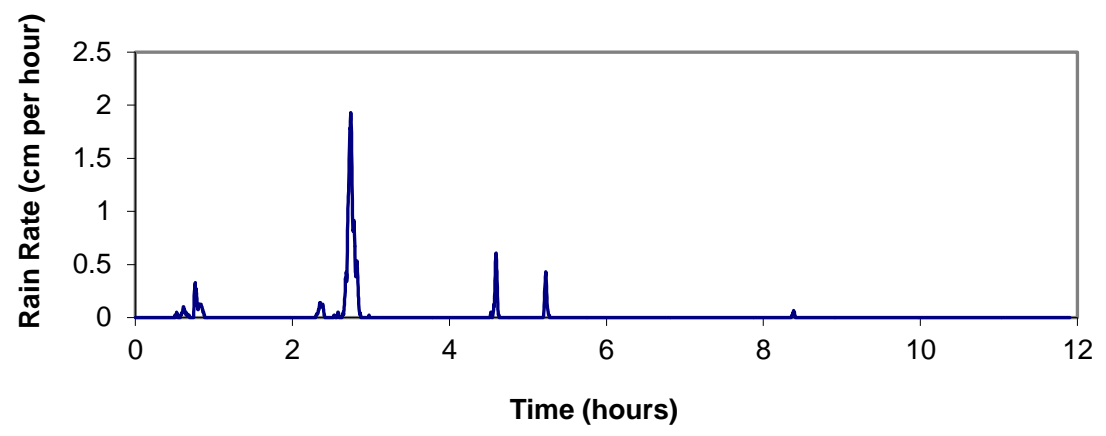

(b)

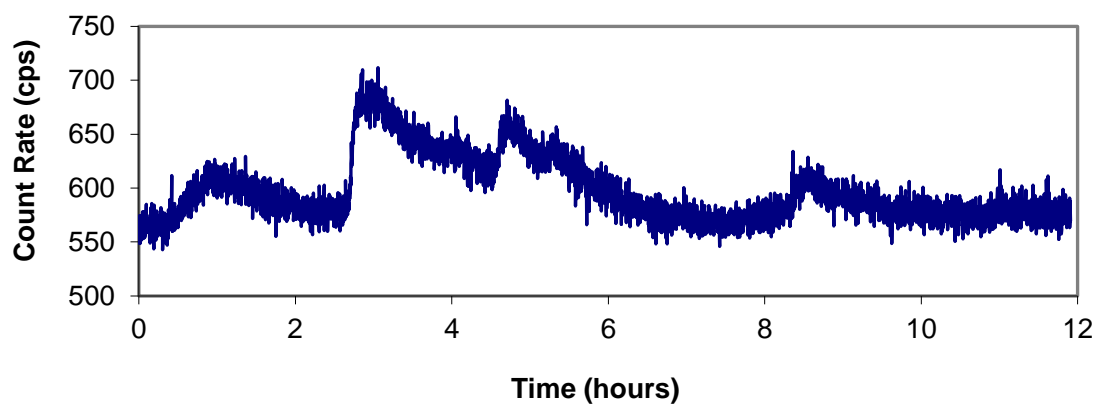

(c)
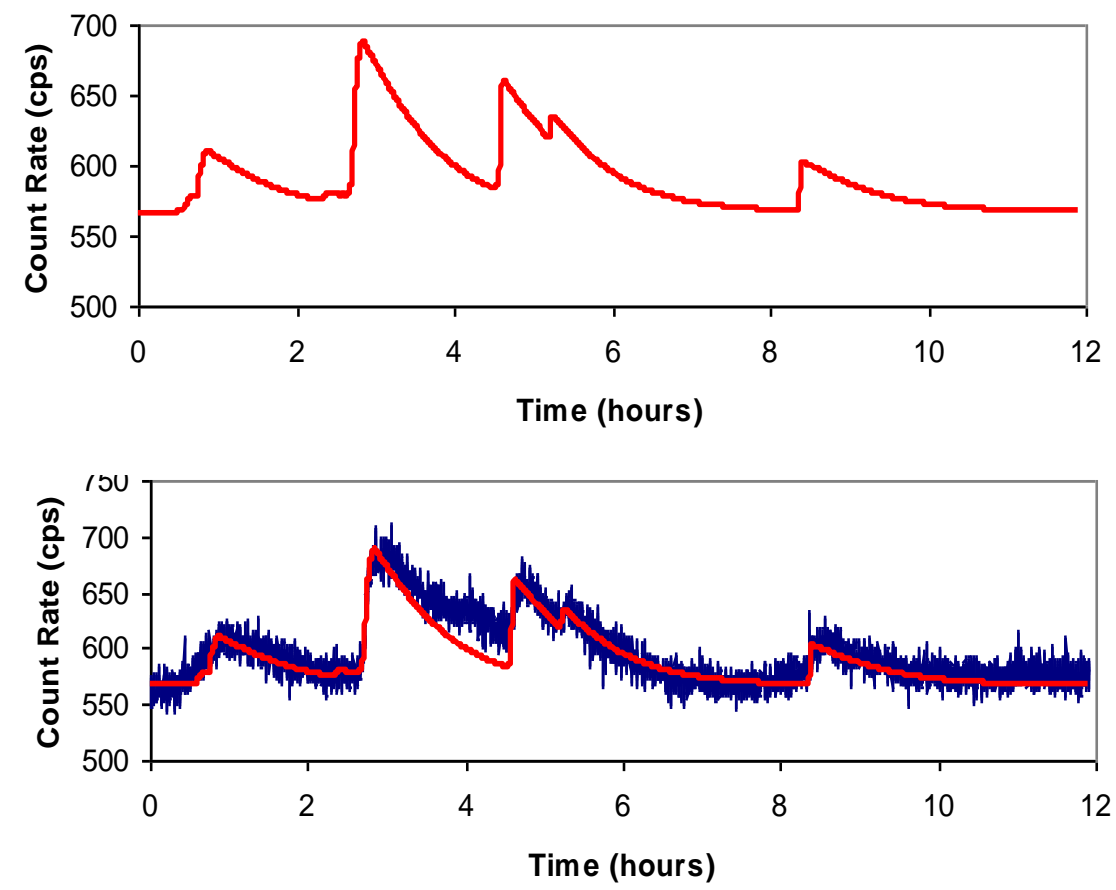

Figure 9: (a) rate of rain in $\mathrm{cm} / \mathrm{hr}$ for an isolated rain event, (b) RPM response to the rain, (c) mathematically predicted response, (d) agreement between the actual RPM response and the predicted response 


\section{Activity Density of Rain}

The amount of radioactivity in each unit of rain greatly varies between rain events and is referred to as the "activity density of rain". To determine the amplitude of the predicted RPM response, the activity density for each independent rain event must be determined. The amount of ${ }^{214} \mathrm{~Pb}$ and ${ }^{214} \mathrm{Bi}$ in rain is dependent on a variety of factors and the precise influence of these factors on activity density is not well understood [8]. Consequently, the activity density of each rain event must be measured by comparing the rain intensity and the RPM response.

The activity density of each rain event can be calculated by performing a chi-square goodness of fit test over the recorded data. This will result in a sample of activity densities that are used to analyze the relationship between activity density and various environmental conditions. Environmental conditions that will be studied in the future included wind direction, rain intensity, rain duration, temperature, and season.

Understanding the activity density of rain and how it is affected by environmental conditions is necessary for predicting the RPM response due to rainfall. Different locations exhibit varying activity densities so measurements need to be performed at each potential RPM site. The collection of this data will not only yield the expected RPM background of a site; it will also contribute to a greater study of rain activity densities and their environmental influences.

\section{Future Work}

The future of this work will take several directions. Firstly, the mitigation of the effects of increased background due to rain will be specifically addressed. It is expected that a roof covering the entire RPM will help, particularly if coupled with good drainage. There will also be studies of different shielding configurations, to determine economical, effective solutions. These solutions will be compared to calculations, so that the individual components of the background are isolated and understood.

Secondly, the radiation and weather measurement system will be modularized for background studies in remote locations. A portable system for the study of radioactive precipitation will allow further research on the environmental factors that affect precipitation activity density. For instance, researching the correlation between wind direction and activity density is expected to allow the verification of source material for cloud-radioactivity formation.

\section{Conclusions}

There are examples of RPM installations, where the rain-induced increase of background reaches $300 \%$ its nominal value. Increasing background decreases system sensitivity, and in the case of rain (or other transient phenomena) the sensitivity will fluctuate over the duration of the event.

Studies of RPM response to rain-induced increases of background radiation are found to be consistent with established mechanisms. Time-correlated data from a RPM, HPGe, and a 
weather station proved the increase in background recorded on RPMs during precipitation is a result of the deposition of radon progeny on the ground. After limiting the RPM response to the decay of radon progeny, a method was developed for fitting complicated RPM behavior by allowing each rain event to be independently normalized. Predicting the RPM response to a rain event allows the analysis of potential RPM sites to determine the background the RPMs will experience. Rain-deposited activity can be treated in much the same way that radioactive paving materials are treated, by shielding and collimating the detectors so they have a limited view of the activity. In addition, by obtaining a fundamental understanding of how precipitation increases the background of RPMs, data from RPMs around the world can be utilized to track the activity density of rain and conduct further research in the area of environmental cloud science.

\section{References}

[1] J. F. Mercier, et. al., "Increased environmental gamma-ray dose rate during precipitation: a strong correlation with contributing air mass", Journal of Environmental Radioactivity 100 (2009): $527-533$.

[2] M. B. Greenfield, et. al., "Determination of rain age via $\gamma$ rays from accreted radon progeny", Journal of Applied Physics 104 (2008) 074912.

[3] Radon Transport through and Exhalation from Building Materials. U.S. Department of Commerce / National Bureau of Standards, NBS Technical Note 1139, September 1981.

[4] Introductory Nuclear Physics, Kenneth S. Krane, John Wiley and Sons, 1987

[5] Environmental Radioactivity (from Natural, Industrial, and Military Sources), $3^{\text {rd }}$ Edition, Merrill Eisenbud. Academic Press Inc, 1987

[6] Atmospheric Diffusion (the Dispersion of Windborne Material from Industrial and other Sources), F. Pasquill, D. Van Nostrand Company Ltd., 1962

[7] Concentrations and their ratio of $222 \mathrm{Rn}$ decay products in rainwater measured by gamma-ray spectrometry using low-background Ge detector, Masanori Takeyasu et.al., Journal of Environmental Radioactivity 88 (2006) 74-89.

[8] Cortes, Guillem, Joseph Sempau, and Xavier Ortega. "Automated measurement of radon daughters Bi-214 and Pb-214 in rainwater." Nukleonika. 46.4 (2001): 161-164.

[9] Fujinami, Naoto. "Observational Study of the Scavenging of Radon Daughters by Precipitation from the Atmosphere." Environmental International. 22.1 (1996): 181-185. 